\title{
Modelling the Performance of Single-Photon Counting Kinetic Inductance Detectors
}

\author{
Josie Dzifa Akua Parrianen ${ }^{1}$. Andreas Papageorgiou ${ }^{1}$ - Simon Doyle ${ }^{1}$. \\ Enzo Pascale ${ }^{1,2}$
}

Received: 6 November 2017 / Accepted: 28 August 2018 / Published online: 1 September 2018

(c) The Author(s) 2018

\begin{abstract}
We present the first published results of near-infrared single-photon detection in aluminium lumped element kinetic inductance detectors (LEKIDs). Using aluminium as a well-understood material that follows conventional superconductor theory, we discuss and validate a model that describes the energy-resolving performance of a LEKID to single-photon absorption events. We also discuss data analysis techniques used to extract single-photon detections from noisy data. We measure an energy resolution of $662 \mathrm{meV}$ for a $1550 \mathrm{~nm}$ photon source which is in close agreement to our model predictions for this non-optimised device limited by generation-recombination noise.
\end{abstract}

Keywords Single photon · Energy resolving · Kinetic inductance detectors

\section{Introduction}

A principal goal of the next generation of space-based astronomy will be dedicated to the characterisation of extra-solar planets (exoplanets). Of the 1000s discovered, only a handful have been spectrally analysed. As such, there is increasing interest in new exoplanet missions aiming to carry out spectroscopy on very low intensity light and shallow transit light curves. Energy-resolving, single-photon counting detectors provide an elegant solution for carrying out imaging spectroscopy without the need for gratings, prisms or combinations thereof. Kinetic inductance detectors (KIDs) are a proven technology capable of energy-resolving single-photon events at optical and near-infrared (NIR) wavelengths. Recent experiments such as ARCONS an optical to NIR spectrophotometer [1] have achieved energy resolutions of order $300 \mathrm{meV}$ at $400 \mathrm{~nm}$. However, this is $\sim 10$ times worse than the theoretical limit imposed by

\footnotetext{
$凶$ Josie Dzifa Akua Parrianen josie.parrianen@astro.cf.ac.uk

1 School of Physics and Astronomy, Cardiff University, Cardiff, UK

2 Dipartimento di Fisica, Sapienza Universita di Roma, Rome, Italy
} 
Poisson statistics. Similar experiments working at $1550 \mathrm{~nm}$ show the same deviation from theoretical limits achieving energy resolutions of order $220 \mathrm{meV}$ [2]. Still, this is just a factor of $\sim 2$ worse than transition edge detectors in the same regime [3, 4]. Here we present the first published results of NIR single-photon response in aluminium (Al) LEKIDs.

\section{Single-Photon Response Model}

KIDs are thin-film, superconducting microresonators [5]. Photon absorption leads to the breaking of Cooper pairs to create quasiparticles (un-paired electrons in the superconductor). The quasiparticle population determines the resonance frequency, of the resonator, through its surface impedance. Changes to the surface impedance are governed by kinetic inductance.

The absorption of a photon of energy $h v$ creates a proportional number of excess quasiparticles; we assume the population is increased uniformly across the detector. A first-order estimate is given by $N_{\mathrm{qp}, x s}=\eta h v / \Delta$, where we assume $\eta=0.4$ is the quasiparticle generation efficiency for thin-film $\mathrm{Al}[6]$ and $\Delta$ is the superconductor energy gap. Any change in the quasiparticle population corresponds to a change in the detector's resonance frequency $d f_{0}$. Using conventional superconductivity theory, we simulate the detector response as a function of change in quasiparticle population $d f_{0} / d N_{\mathrm{qp}}$ [7] to find the resonance frequency shift: $d f_{0, \max }=N_{\mathrm{qp}, x s} \times d f_{0} / d N_{\mathrm{qp}}$.

The expected response to single-photon absorption is a pulse with an exponential decay of the resonance frequency shift $d f_{0}$. The decay time is governed by the dominant time constant of the detector. We assume this to be the quasiparticle lifetime $\tau_{\mathrm{qp}}$, such that $d f_{0}(t)=d f_{0, \max } e^{-t / \tau_{\mathrm{qp}}}$. Note that $\tau_{\mathrm{qp}}$ is longer than the detector ring-up/ringdown time given by $Q / \pi f_{0}$. This is demonstrated in Fig. 2 where the rise time of the pulse is determined by the resonator ring-up time and the far slower decay is determined by $\tau_{\mathrm{qp}}$.

This first-order model assumes that single-photon absorption results in a uniform rise in the quasiparticle distribution across the detector's meander, producing an expected maximum pulse height of $\sim 120 \mathrm{~Hz}$.

\section{Energy Resolution}

The energy resolution of a single-photon detector is given by

$$
\Delta E=h v \times \frac{\text { noise }}{\text { signal }}
$$

In a KID, the noise equivalent power (NEP) for a given detector volume and temperature is fundamentally limited by the random fluctuation in the quasiparticle 
population, known as generation-recombination (GR) noise. This limit is described by [8]

$$
\mathrm{NEP}_{\mathrm{GR}}=2 \Delta \sqrt{N_{\mathrm{qp}} / \tau_{\mathrm{qp}}}
$$

We multiply Eq. (2) by the detector time constant $\tau_{\mathrm{qp}}$ to convert to noise equivalent energy $\mathrm{NEE}_{\mathrm{GR}}=2 \Delta \sqrt{N_{\mathrm{qp}} \tau_{\mathrm{qp}}}$, which gives the energy resolution in $0.5 \mathrm{~s}$ of integration time. For single-photon events, the response is measured over the detector time constant; in our case, it is dictated by $\tau_{\mathrm{qp}}$. Taking into consideration the quasiparticle generation efficiency, the GR noise-limited energy resolution is approximated by

$$
\sigma_{\mathrm{GR}}=\frac{\Delta}{\eta} \sqrt{2 N_{\mathrm{qp}}} .
$$

The fundamental noise for a detector capable of single-photon detection is governed by the Fano limit and is given by

$$
\sigma_{\text {photon }}=\sqrt{\frac{h v F \Delta}{\eta}},
$$

where $F=0.2$ is the Fano factor [9]. For a KID, the Fano factor takes into account the variance in the number of quasiparticles generated due to single-photon absorption events.

We therefore propose a limit on the energy resolution of a single-photon KID as a combination of the two fundamental limits from Eqs. (3) and (4). The convention is to quote the full-width-at-half-maximum (FWHM) energy resolution, where FWHM = $2.355 \sigma$ so that

$$
\Delta E_{\lim }=2.355 \sqrt{\frac{\Delta^{2}}{\eta^{2}} 2 N_{\mathrm{qp}}+\frac{h v F \Delta}{\eta}} .
$$

The devices were measured at $100 \mathrm{mK}$ and have meander volumes of $\sim 1400 \mu \mathrm{m}^{3}$. Based on the theory outlined above, we predict $\sigma_{\mathrm{GR}}=1.3 \mathrm{meV}$, and thus, $\Delta E_{\mathrm{lim}}=$ $21 \mathrm{meV}$ for our test devices following Eq. (5). This suggests the detector is at the fundamental photon limit. However, the quasiparticle lifetime (and equivalently the quasiparticle density) has been shown to saturate at some temperature due to microwave heating $[10,11]$. We have measured this saturation in our device, see Fig. 2, showing a saturation temperature of approximately $190 \mathrm{mK}$. This adjusts our GR-limited 1-sigma energy resolution to $\sigma_{\mathrm{GR}}=142 \mathrm{meV}$, and thus, we predict $\Delta E_{\text {lim }}=335 \mathrm{meV}$, putting our detectors very firmly in the GR noise limit.

\section{Experimental Set-Up and Measurement}

We used a 624-pixel lumped element (LE) KID array developed for part of the SpaceKIDs project [12], optimised as a $350 \mathrm{GHz}$ narrowband Earth observation 

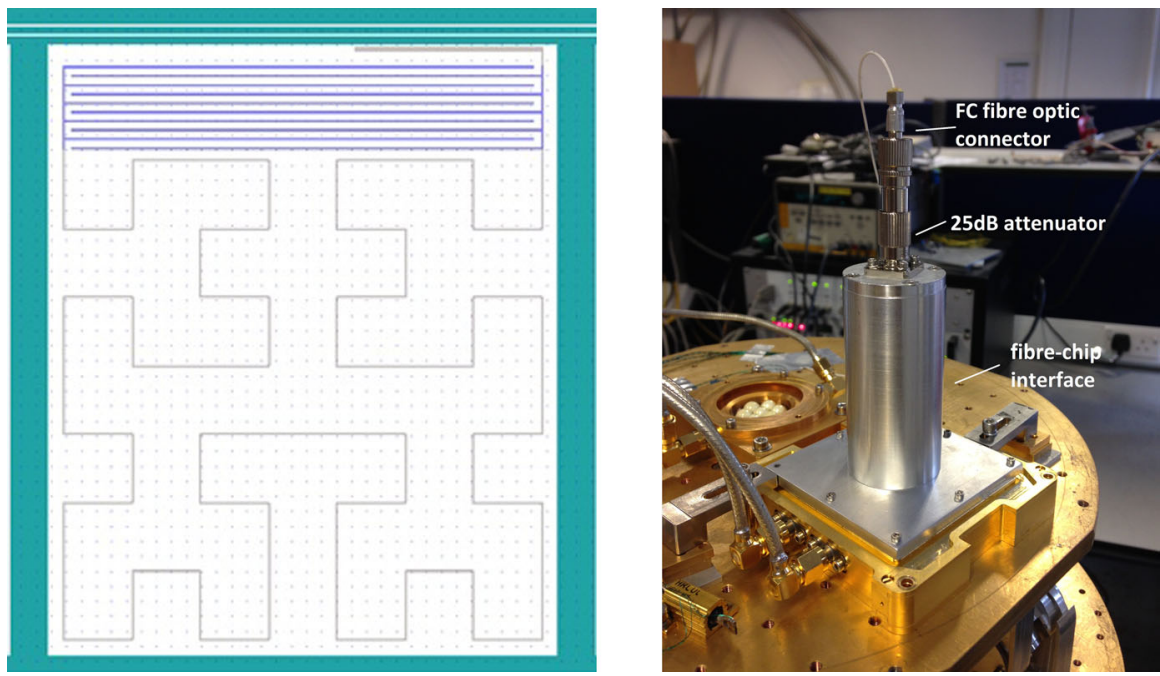

Fig. 1 Left: Schematic of LEKID architecture. Interdigitated capacitor (IDC) and meander have linewidth $4 \mu \mathrm{m}$ with meander volume $\sim 1400 \mu \mathrm{m}^{3}$. Right: Image of fibre-chip interface (Colour figure online)

demonstrator. The array is formed of a $30 \mathrm{~nm} \mathrm{Al} \mathrm{film}\left(T_{\mathrm{C}}=1.3 \mathrm{~K}\right)$ on a $320-\mu \mathrm{m}$ silicon ( $\mathrm{Si}$ ) substrate. There is an additional low $T_{\mathrm{C}}$ titanium aluminium (TiAl) bilayer on the backside of the substrate, designed as a phonon-absorbing layer to limit the effects of cosmic ray events in the array. The pixel design follows standard LEKID architecture [13] with the inductive meander patterned into a third-order Hilbert fractal, shown in Fig. 1.

The array was cooled down, to a base temperature of $100 \mathrm{mK}$, using a miniature dilution refrigerator. A standard homodyne readout technique was used to measure the detector response. Measurements were made with a $1550 \mathrm{~nm}$ laser diode mounted on the $4 \mathrm{~K}$ stage. The incident photons are carried by a $9 \mu \mathrm{m}$ single-mode fibre optic cable up to the ultra-cold stage and are attenuated before entering the device holder. A modified plate, shown in Fig. 1, ensures roughly half of the pixels are directly illuminated. The device is flood illuminated at a constant DC power level. The time-dependent fluctuations in resonant frequency are measured and analysed under illuminated and non-illuminated conditions.

\section{Results}

The analysis of the data produced in this work has two main aims: (1) to measure the impulse response decay time constant $\tau$ and (2) to identify single-photon absorption events and measure the pulse amplitude of such events.

The impulse response time constant was measured by illuminating the detector with optical square-wave pulses and fitting to the pulse decay time. Several pulses were stacked to increase the signal-to-noise ratio (SNR). At temperatures above $200 \mathrm{mK}$, the SNR became too low, at which point we use the method of fitting to the noise 

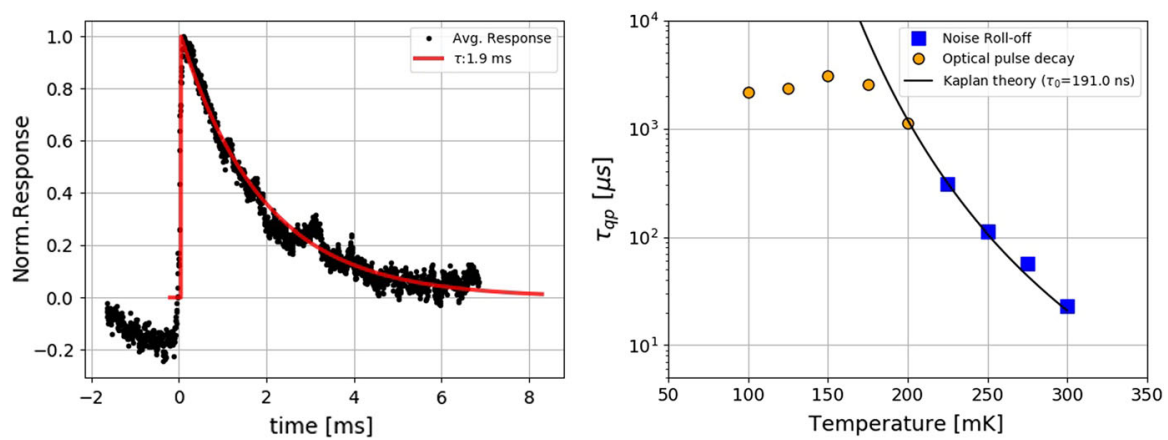

Fig. 2 Left: Averaged normalised stacked impulse response found in the illuminated detector response. An exponential (red line) is fitted with $\tau=1.9 \mathrm{~ms}$. Right: The quasiparticle lifetime as a function of bath temperature, using two methods for extracting the time-constant. Kaplan theory [14] is fitted to the noise roll-off data (blue) (Colour figure online)
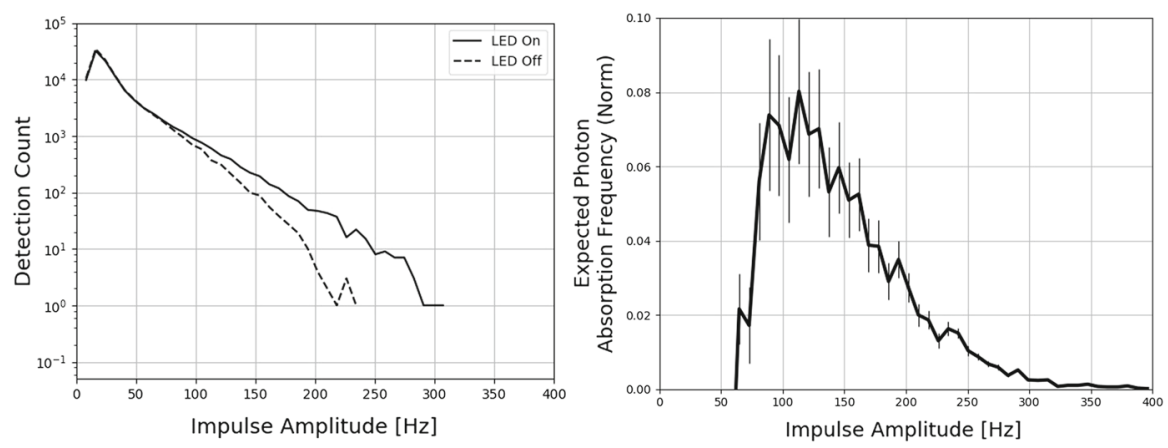

Fig. 3 Left: Impulse detection counts as a function of impulse amplitude; detection counts shown for both dark (LED off) and illuminated (LED on) detector. Dark detections are false detections and represent the sensitivity limits of the detection algorithm. Right: Normalised expected photon absorption event distribution, which shows a peak at approximately $120 \mathrm{~Hz}$

roll-off from the detector response power spectral density (PSD) [10]. Figure 2 shows the combined detector time constant fitting results.

The time constant was validated by fitting to detector impulse responses to singlephoton absorption events. Potential absorption events were identified by a step function matched filter. Such events were stacked and averaged, as shown in Fig. 2, which has been fitted with an exponential of $\tau=1.9 \mathrm{~ms}$, matching the same value found for square-wave measurements made at $100 \mathrm{mK}$. We take this as the quasiparticle lifetime.

Having obtained an estimate of the impulse response, $20 \mathrm{~s}$ response time streams were Wiener filtered - with bandwidth $\sim 2 \mathrm{kHz}$ - and potential photon absorption events were identified by matched filter. The same procedure was applied to both illuminated and dark detector data; the extracted counts are shown in Fig. 3. The dark detector has only "false" events due to the limitations of the impulse extraction procedure, while the illuminated detector counts comprise both "true" and "false" events. The illuminated detections significantly outnumber the false detections, picked out from the dark detector, for impulses of amplitude $>200 \mathrm{~Hz}$. This surplus of detections 
is attributed to true photon absorption events. Given the extraction method employed here, photon absorption events with amplitude $<200 \mathrm{~Hz}$ are more difficult to discern between true and false detections. This uncertainty is depicted by the vertical error bars shown in Fig. 3. Note that counts below $50 \mathrm{~Hz}$ have been set to zero due to excessive noise.

The expected photon absorption distribution is then measured by subtracting the dark data detections from the detections of the illuminated data (using a coefficient of 1), resulting in the plot shown in Fig. 3. Notice the photon absorption distribution peaks at an amplitude of $\sim 120 \mathrm{~Hz}$, matching our expected pulse height. There may also be a peak at $\sim 240 \mathrm{~Hz}$ with very small error bars, suggestive of two-photon absorption events.

As an initial estimate for the energy resolution of our measurements, we refer to Eq. (1), taking our noise as the FWHM of the right plot from Fig. 3 and the peak value as our signal, such that $\Delta E_{\text {calc }}=662 \mathrm{meV}$. This is $\sim 2$ times worse than expected. However, the Weiner filtering process reduces the integration time to $\sim \tau_{\mathrm{qp}} / 4$ leading to a $\sqrt{4}$ increase in GR noise contributions: $\Delta E_{\text {lim }}=668 \mathrm{meV}$. Accounting for the reduced effective integration time, our measured value is in rough agreement with our predicted theoretical limit. Measuring such low energy photons in the presence of relatively high GR noise has resulted in poor SNR (as depicted by the error bars in Fig. 3), with the absorption distribution being not well defined. As such the true peak value is not easily discerned and the Gaussian width used to calculate energy resolution is likely skewed by the apparent $\sim 240-\mathrm{Hz}$ peak. We also note it is likely the distribution includes responses from photons absorbed in the capacitor section of the device where reduced response is observed, as has been seen in similar devices [2].

\section{Conclusions and Future Work}

We show confirmation of single-photon detection which corroborates our first-order sensitivity model in the presence of GR noise, using $\eta=0.4$ for thin-film Al. We have also shown the limiting effects of microwave heating on such devices that complies with theory and measurement. We plan to fully confirm this model by measuring devices of varying volumes over a range of optical frequencies and operating temperatures enabling clear mapping of the Fano and GR noise contributions to energyresolving LEKIDs.

Acknowledgements We acknowledge the Science and Technology Facilities Council (STFC) Consolidated Grant Ref: ST/N000706/1 and studentship funding for supporting this work. We also take the opportunity to thank the reviewers of this paper for their insightful comments.

Open Access This article is distributed under the terms of the Creative Commons Attribution 4.0 International License (http://creativecommons.org/licenses/by/4.0/), which permits unrestricted use, distribution, and reproduction in any medium, provided you give appropriate credit to the original author(s) and the source, provide a link to the Creative Commons license, and indicate if changes were made. 


\section{References}

1. B.A. Mazin et al., Publ. Astron. Soc. Pac. 125(933), 1348-1361 (2013)

2. W. Guo et al., Appl. Phys. Lett. 110(21), 212 (2017)

3. A.E. Lita, A.J. Miller, S.W. Nam, Opt. Express 16(5), 3032-3040 (2008)

4. A.J. Miller, S.W. Nam, J.M. Martinis, Appl. Phys. Lett. 83(4), 791 (2003)

5. P.K. Day et al., Nature 425(6960), 817-821 (2003)

6. T. Guruswamy, D.J. Goldie, S. Withington, Supercond. Sci. Technol. 27(5), 55 (2014)

7. S.M. Doyle, Lumped Element Kinetic Inductance Detectors (Cardiff University, Cardiff, 2008)

8. A.V. Sergeev, V.V. Mitin, B.S. Karasik, Appl. Phys. Lett. 80(5), 817-819 (2002)

9. U. Fano, Phys. Rev. 72(1), 26-29 (1947)

10. P.J. de Visser et al., J. Low Temp. Phys. 167(3-4), 335-340 (2012)

11. P.J. de Visser et al., Phys. Rev. Lett. 112(4), 047004 (2014)

12. Griffin, M., et al., SPACEKIDS: kinetic inductance detectors for space applications, in Proceedings of SPIE-The International Society for Optical Engineering (2016)

13. S. Doyle et al., J. Low Temp. Phys. 151(1-2), 530-536 (2008)

14. S.B. Kaplan et al., Phys. Rev. B 14(11), 4854-4873 (1976) 